\title{
Evaluation of gait performance of knee osteoarthritis patients after total knee arthroplasty with different assistive devices
}

\author{
Ana Tereso, Maria Manuel Martins*, Cristina Peixoto Santos
}

\begin{abstract}
Introduction: Nowadays Knee Osteoarthritis (KOA) affects a large percentage of the elderly, and one solution is to perform a Total Knee Arthroplasty (TKA). In this paper, one intends to study the gait and posture of these patients after the TKA, while walking with three assistive devices (ADs) (crutches, standard walker (SW) and rollator with forearm supports (RFS)). Methods: Eleven patients were evaluated in 2 phases: 5 days and 15 days after surgery. This evaluation was conducted with two inertial sensors, one attached to the operated leg ankle, to measure spatiotemporal parameters, and the other at the sacrum, to measure posture and fall risk-related parameters. Multivariate analysis of variance (MANOVA) with repeated measures was performed to detect group differences. Results: The MANOVA results show that all spatiotemporal parameters are significantly different $(p<0.05)$ between the two phases of study. So, time has a significant influence on such parameters. In relation to the ADs, one observes that there are statistical significant effects on all spatiotemporal parameters, except for swing duration and step length $(\mathrm{p}>0.05)$. The interaction between time and ADs only affects significantly the velocity $(p<0.05)$. In terms of fall risk parameters, time only significantly affects the antero-posterior direction $(\mathrm{p}<0.05)$ and $\mathrm{ADs}$ affects significantly root mean square in medio-lateral direction $(\mathrm{p}<0.05)$. In terms of interaction between time and ADs, there are no statistical significant differences. Conclusion: This study concludes that depending on the state of recovery of the patient, different ADs should be prescribed. On the overall, standard walker is good to give stability to the patient and RFS allows the patient to present a gait pattern closer to a natural gait.
\end{abstract}

Keywords: Knee osteoarthritis, Total knee arthroplasty, Assistive devices, Elderly, Walkers.

\section{Introduction}

Osteoarthritis (OA) affects the joints responsible for supporting the body weight, such as the knee, producing restrictions on movement in patients usually over 65 years (Braddom, 2006). Typically, these patients present slow gait and short step length (Debi et al., 2011). Total Knee Arthroplasty (TKA) is a successful surgical procedure to relieve knee OA that results in an improvement in the functional capacity of patients. After 5 days (first phase) of surgery, patients leave bed and start to use the wheelchair. Then, after 15 days (second phase) patient already have the capability of walking more independently, with the help of crutches, as a precaution. Usually, these patients' recovery is made with the help of crutches; however this device might not be the correct one for these patients, since some of them feel knee pain. Since in the second phase of recovery the patients are in an advanced stage of mobility, feeling almost no pain on the knee, the authors decided to compare their first phase gait pattern with their second phase gait pattern, when using different assistive devices (ADs). With this comparison, one intends to infer between different ADs (crutches, standard walker and rollator with forearm supports) which one would have more capability for providing the required support and comfort for post-surgical TKA patients. Thus, if both data sets (first and second phase) of gait measurements do not differ significantly with a specific device, it could mean that the necessary help and functional compensation is being provided by the device. This will open the possibility of substituting, or not, the crutches by other devices for the recovery of post-surgical TKA patients.

Therefore, on this study, spatiotemporal and posture related measurements will be calculated and compared between $3 \mathrm{ADs}$, in two different phases of recovery, through an objective assessment by means of inertial sensors and, finally, compared with healthy people measurements.

Studies about rollators revealed that these devices are safe and stable, providing an increase in confidence, as well as an improvement in balance and mobility (Kegelmeyer et al., 2013; Liu, 2009; Vogt et al., 2010). This $\mathrm{AD}$ also causes a lower variability in gait and it is easy to use (Kloos et al., 2012; Vogt et al., 2010; Wellmon et al., 2006). On the other hand, there are 
several authors indicating that this $\mathrm{AD}$ causes changes in posture and, an increased risk of fall (Kegelmeyer et al., 2013; Liu et al., 2009; Vogt et al., 2010). However, on this study forearm supports were added to the device in order to give more stability to the gait, better posture and increased support (Ishikura, 2001; Martins et al., 2013; Youdas et al., 2005). One problem that could arise is the upper limbs excessive effort (Tung et al., 2014), however since this device will just be used during recovery, i.e. a short time interval, upper limb problems will not be a concern. On the other hand, standard walker (SW) is known as the more stable device, supporting a greater percentage of body weight (Melis et al., 1999). Still, it provides a slow and varied gait, less mobility with higher metabolic cost due to reduced speed and repetitive motion for lifting it while moving forward (Kegelmeyer et al., 2013; Kloos et al., 2012; Melis et al., 1999; Priebe and Kram, 2011; Roomi et al., 1998). Crutches have upper limbs support and enable an easy climbing of the stairs (Van Hook et al., 2003). However, these devices require some energy cost, excessive upper limb effort, balance problems and do not provide for a natural gait (Bradley and Hernandez, 2011).

Therefore, it is expected that rollator with forearm supports (RFS) will provide more support for the gait, decreasing knee pain, provide a higher velocity and cadence and a more natural gait pattern for the TKA patients. In contrast, SW will provide a better balance for the patients.

The aim of this study is to assess which ADs have the capability of providing the necessary compensation to the TKA patients after surgery. This conclusion will be assessed depending on the results of the statistical analysis of two sets of parameters, i.e. spatiotemporal parameters and posture and fall-risk measurements.

\section{Methods}

\section{Participants}

A group ( $\mathrm{N}=11)$ of elder patients ( 3 men and 8 women) aged $67.3 \pm 5.1$ years diagnosed with osteoarthritis in the knee and subjected to TKA was considered. These people do not have experience using SW and RFS, since during their recovery they only use crutches. The inclusion criteria were patients on the fifth day after TKA, hemodynamically stable that have already lift from the bed, with cognitive capabilities, presenting flexion of some muscles of the hip, wrist, elbow and knee and BERG test score less than 45 . The exclusion criteria were cardiac, vascular, respiratory, neurologic or metabolic disease that affect the gait; neurologic disease that affects the balance; pathology in the ear and recent clinical history of trauma in the limbs. The study was conducted at Braga Hospital, approved by the Ethical Committee, and all the patients signed the informed consent.

\section{Protocol and parameters}

In order to assess the effect of the ADs on gait, tests were conducted using two crutches, SW, and RFS (Melis et al., 1999), showed in Figure 1. The crutches are two metal vertical props that extend from the walking surface to the arm. The SW has a basic design that consists of a lightweight frame that is about waist high, approximately $30 \mathrm{~cm}$ deep and slightly wider than the user. It has four rubber end points for a better support on the ground. The RFS consists on a frame with four wheels, handlebars, forearm supports and
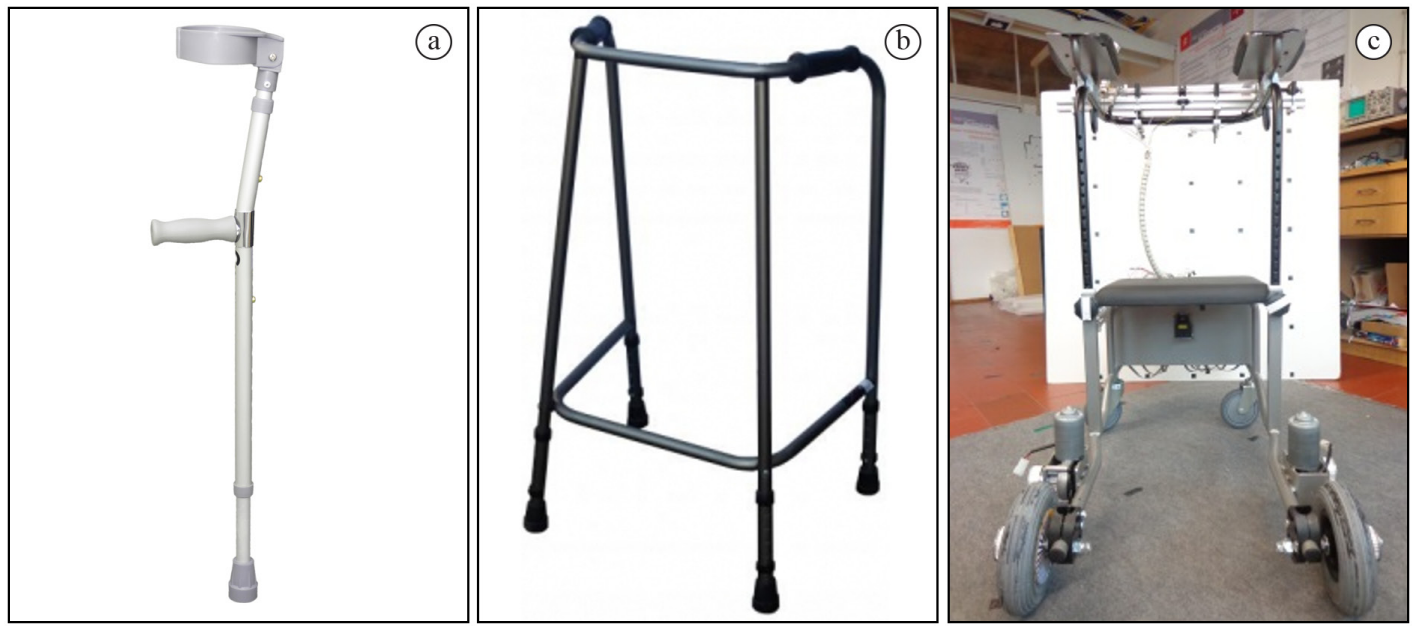

Figure 1. a) Crutches; b) Standard walker; c) RFS walker 
a built-in seat, which allows the user to stop and rest when needed. It is adjustable in height.

Tests consisted on subjects walking straight forward approximately $10 \mathrm{~m}$ with the $3 \mathrm{ADs}$, along a corridor at the Hospital of Braga. Three walking trials for each subject and condition are performed. All trials and tests are filmed by a video camera, in order to verify sensor error situations. The mean and standard deviation are computed for each parameter. Before performing the tests, a therapist adjusted the height of each AD for each subject. To measure the spatiotemporal and posture parameters, two 3-axis inertial sensors are used. These sensors (SMI, MP6000 of InvenSense) need a computer and a base station (CC2530 of Texas Instrument). In this study only the accelerometer is used. One sensor is attached to the operated leg ankle, to measure spatiotemporal parameters, and the other at the sacrum (trunk), to measure posture and fall risk-related parameters. The used system configuration and the coordinates of reference are shown in Figure 2. The $\mathrm{x}$-axis, $\mathrm{y}$-axis and $\mathrm{z}$-axis correspond to the medio-lateral (ML), vertical (V) and anterior-posterior (AP) accelerations, respectively. The spatiotemporal parameters considered for this study are: stride time (TStride), swing (TSwing) and stance (TStance) phases, velocity, cadence and step length $(S L)$. The obtained spatiotemporal values will be compared with healthy elderly values calculated during free walking extracted from Hollman et al. (2011) study.

The posture parameters are the root mean square of AP and ML acceleration (RMSAP and RMSML), Range of motion of AP and ML directions (ROMAP and $R O M M L$ ) and horizontal displacement of Centre of Mass (DCOM). The data processing of the accelerometer signals and respective calculation of the presented parameters is explained in detail at Tereso et al. (2014) and on the following subsection.

The obtained posture parameters will be compared with the same parameters derived from a study conducted at University of Minho, with eight healthy young men. The tests with the patients consisted of walking free in a straight line with $10 \mathrm{~m}$.

\section{Data acquisition and processing}

The calibration of the accelerometer and previous signal processing are described in Fernandes (2013). The data acquired in each trial is saved in the SD
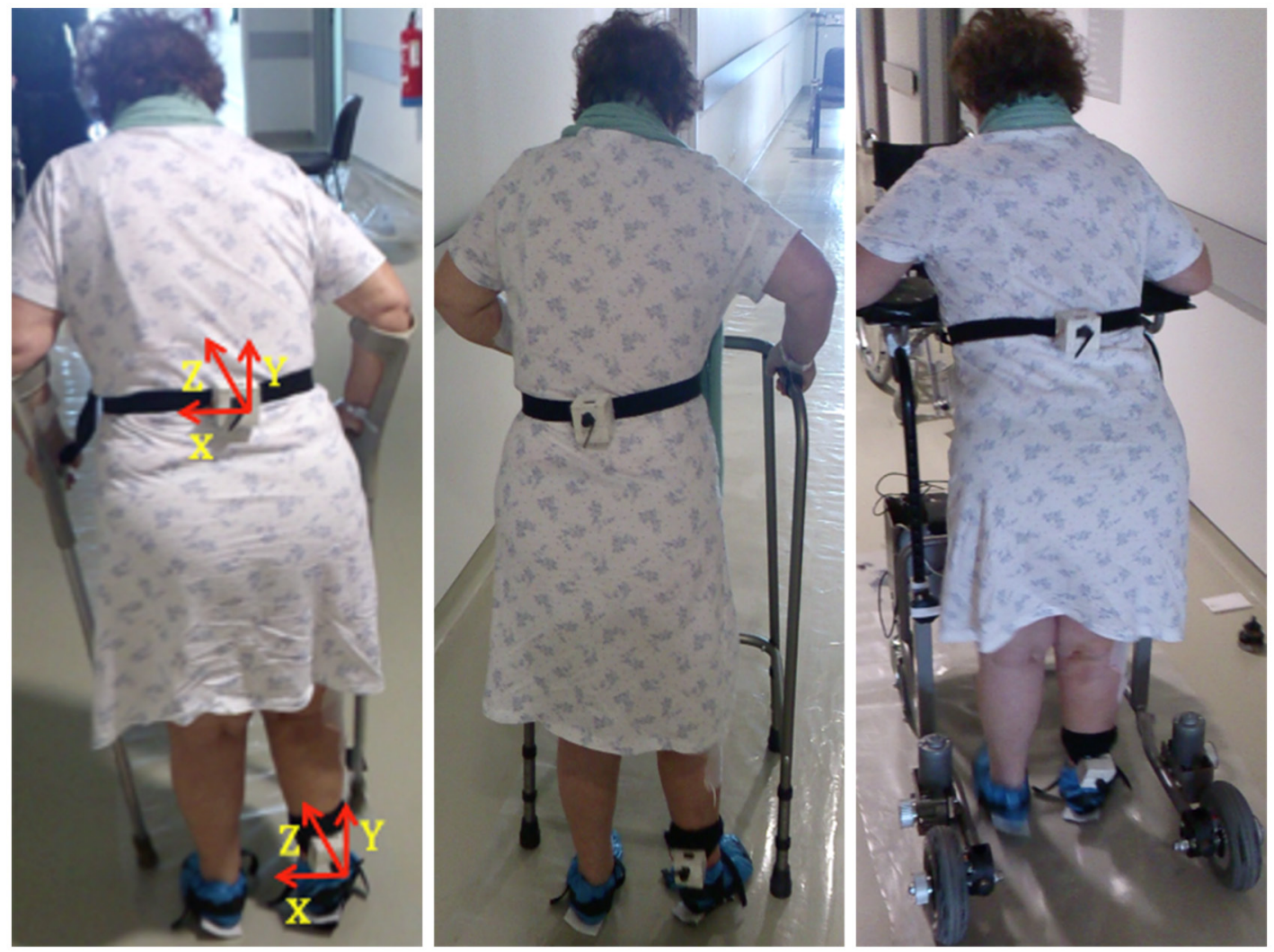

Figure 2. Example of the experimental setup with the assistive devices used in this study. Left: Crutches (orientation of the sensors axes indicated); Center: standard walker; Right: RFS walker. 
card of the sensor, read at the $\mathrm{PC}$ and processed on MATLAB 2013.

The algorithm implemented in this study for the detection of gait events (heel strike and toe-off) is based on (Lee et al., 2010). These two events are essential for the calculation of gait parameters like stance and swing phase.

The implementation consists on the detection of the time peak of Heel Strike (HS) and Toe-Off (TO) events. First, at each instant of time, the data of each axis is summed and transformed to produce the 'Signal Vector Machine', represented by s:

$s=\sqrt{a_{x}^{2}+a_{y}^{2}+a_{z}^{2}}$

where $a_{x}, a_{v}$ and $a_{z}$ are the ML, V and AP accelerations, respectively.

This step is applied since acceleration is highly influenced by the position of the sensor and the 3 axis have significant information. Second, $s$ is filtered by a low pass filter $\left(\mathrm{f}_{\text {pass }}=6 \mathrm{~Hz}, \mathrm{f}_{\text {stop }}=10 \mathrm{~Hz}\right)$ to extract features related to the gait cycle,

$$
y[n]=\sum_{i=0}^{10} b_{i} s[n]
$$

where $b_{i}$ corresponds to the coefficients of the filter. These coefficients are obtained by running the fdatools interface in MATLAB. Third, a least-square polynomial derivative approximation filter eliminates noise (points that could be considered wrongly as peaks),

$$
z[n]=\frac{1}{10}(1 y[n]+y[n-1]-y[n-3]-2 y[n-4])
$$

After this processing, the final step consists on the peak detection. For each gait cycle there are two peaks, each of them corresponding to a gait event (HS and TO). Before this last step, it was necessary to remove some sample points from the start and end of each test, which correspond to the period of acceleration and deceleration in gait, respectively. The duration of these periods is irregular.

After detecting both events, some gait parameters can be calculated. The gait cycle is divided in two phases - stance and swing. The majority of the gait cycle is spent in stance phase $(60 \%)$ and the rest in swing phase $(40 \%)$. The stance phase corresponds to the moment that the foot is in contact with the ground. The swing phase is the period during which the leg is out of the ground, moving to the next strike. Thereby, stance phase begins with HS event and finishes with TO event. Swing phase begins with TO and finishes with HS. Once stance and swing phases are detected, stride time, cadence, average velocity and step length gait parameters can be calculated. With reference to the $\mathrm{i}$-th gait cycle, the parameters are calculated as follows (Henriksen et al., 2004; Sabatini et al., 2005).

Stride Time $(S T)[s]$ :

$S T(i)=T_{H S}(i+1)-T_{H S}(i)$

Duration of the Swing phase $(S g T)[s]$ :

$\operatorname{Sg} T(i)=T_{H S}(i+1)-T_{T O}(i)$

Duration of the Stance phase $(S t T)[s]$ :

$S t T(i)=S T(i)-S g T(i)$

Cadence $(C)[$ steps/min $]$ :

$C=\frac{\text { Nsteps }}{\text { TotalTimeSteps }}$

The number of steps and the total time corresponding to the number of steps considered was determined by observation of the video.

Average Velocity $(v)[\mathrm{m} / \mathrm{s}]$ :

$v=\frac{\text { Dist }}{\text { TotalTime }}$

The distance (Dist) was measured by tape and the total time corresponds to the duration of the all trials.

Average step length $(S L)[m]$ :

$S L=\frac{v}{C a d}$

The risk of fall of the subject is assessed by an accelerometer attached to the trunk. All the processing applied in these signals is adapted from (Doheny et al., 2002). A $0.1-10 \mathrm{~Hz}$ band-pass filter of fifth order was used to restrict remove artifacts. Then, to obtain the displacements of the subject's COM (DCOM) the acceleration signal (ACC) is double integrated, using a trapezoidal method.

$A C C=\sqrt{A c c M L^{2}+A c c A P^{2}}$

The error associated to the integration (low frequency drift) is reduced by subtracting the mean of the acceleration signals before and after each integration, and then implementing a second-order polynomial fit and a high-pass filter of fifth order of $0.1 \mathrm{~Hz}$.

Displacement ML and AP were also obtained through double integration of the acceleration in ML and AP directions. These signals enable to determine the Root Mean Square (RMS) and Range of motion (ROM) for AP and ML directions (Range of motion (RMSML, RMSAP, ROMML and ROMAP)). 
These parameters enable to assess the fall-risk of the patient. Their values state if the fall-risk is high or not. The higher the values the higher the risk of fall (Doheny et al., 2002; Huisinga et al., 2011).

\section{Statistical analysis}

The mean and standard deviation of each parameter is calculated. Then, an exploratory analysis is made to infer the normality of data. A MANOVA (Multivariate Analysis of Variance) with repeated measures is performed since more than two dependent variables (spatiotemporal and fall-risk parameters) are evaluated in two phases of time (5 days and 15 days). The independent variable is expressed as Time. The tests are performed with three ADs (crutches, SW and RFS), which are the levels of the independent variable or factors considered in MANOVA model. This statistical analysis allows determining if different levels of the independent variable have significant effects on the dependent variables (interaction), what are the interactions among the dependent variables (within subjects) and among the independent variables (between subjects).

Additionally, post hoc Tukey tests are performed to determine, for each dependent variable, which combinations of ADs show significant differences. In all statistical analysis, the significance level was set at 0.05 . The software used was SPSS (version 22).

\section{Results}

The MANOVA results ( $p$ values) for the two data sets are presented in Table 1 and Table 2.

Through observation of Table 1 , one verifies that between phases there is a significant difference on all parameters $(p<0.05)$. Time has a significant influence on TStride, TSwing, TStance, Velocity, Cadence, and $S L(p<0.05)$. In relation to the ADs, one observes that there are statistical significant effects on all parameters as TStride, TStance, Velocity and Cadence $(p<0.05)$, except for TSwing and $S L(p>0.05)$. The interaction between time and ADs (time*factor) only affects significantly the velocity $(p<0.05)$ and not significantly TStride, TSwing, Stance, Cadence, and $S L(p>0.05)$. Finally, Tukey post hoc tests results demonstrate that between ADs, one can differentiate RFS' TStride, TStance, Velocity and Cadence values from Crutches/SW.

Table 2 shows that time only significantly affects ROMAP, RMSAP $(p<0.05)$ and not significantly $R M S M L$, and $D C O M(p>0.05)$. The ADs (factor) affect significantly $R M S M L(p<0.05)$ and not significantly ROMAP, RMSAP and DCOM ( $p>0.05)$. In terms of interaction between time and ADs, there are no statistical significant differences (ROMAP, RMSAP, $R M S M L$ and $D C O M(p>0.05))$. Therefore, the Tukey post hoc tests indicate the existence of significant differences between the RFS and the remaining ADs only for $R M S M L$.

Tables 3 and 4 present the mean and standard deviation of the two parameters data sets: 5 days (parameters with "5") and 15 days (parameters with "15"). Reference values for the parameters taken from Hollman et al. (2011) are also presented.

In general (Table 3), at 5 days, the RFS provides for a faster gait - higher speed and cadence, shorter stride time, stance and swing duration, higher percentage of the swing phase and lower percentage of stance phase. The other ADs are associated with a slower gait, and crutches present a higher step length. Regarding the parameters at 15 days, RFS is still the one that provides the fastest gait, while SW is associated with the slowest gait.

In terms of Table 4, at 5 days, the highest and lowest amplitudes are associated with SW and RFS, respectively. While at 15 days, the highest amplitude corresponds to RFS and the lowest to SW.

\section{Discussion}

\section{Study of spatiotemporal parameters}

This study indicates that all spatiotemporal parameters present significant differences between phases of recovery (Table 1). These differences can be related to a progression or regression during the recovery of the studied patients. Comparing the values of the spatiotemporal parameters of TKA patients with the values obtained with healthy elder subjects (Hollman et al., 2011), an approximation is observed, which means that the evolution of these parameters in TKA patients is going in the direction of improvement of the recovery.

Each AD has a significant different effect on the measured spatiotemporal parameters, independently of the phase at which each patient is, allowing the distinction between them (AD), for each parameter. Only $S L$ and $T S$ wing present no significant differences among ADs (Table 1), since they have similar values among the different ADs (Table 3). The remaining parameters are influenced by the different ADs because of the different type of gait that is adopted by the patient. Regarding the crutches, the patient learns a three points gait, i.e. he always has one foot and two crutches on the ground while moving forward (Smidt and Mommens, 1980). With SW, patients first lift and move the device, placing it in front of them and then they move their legs. Finally with RFS, the movement is continuous by pushing the walker. It is believed 


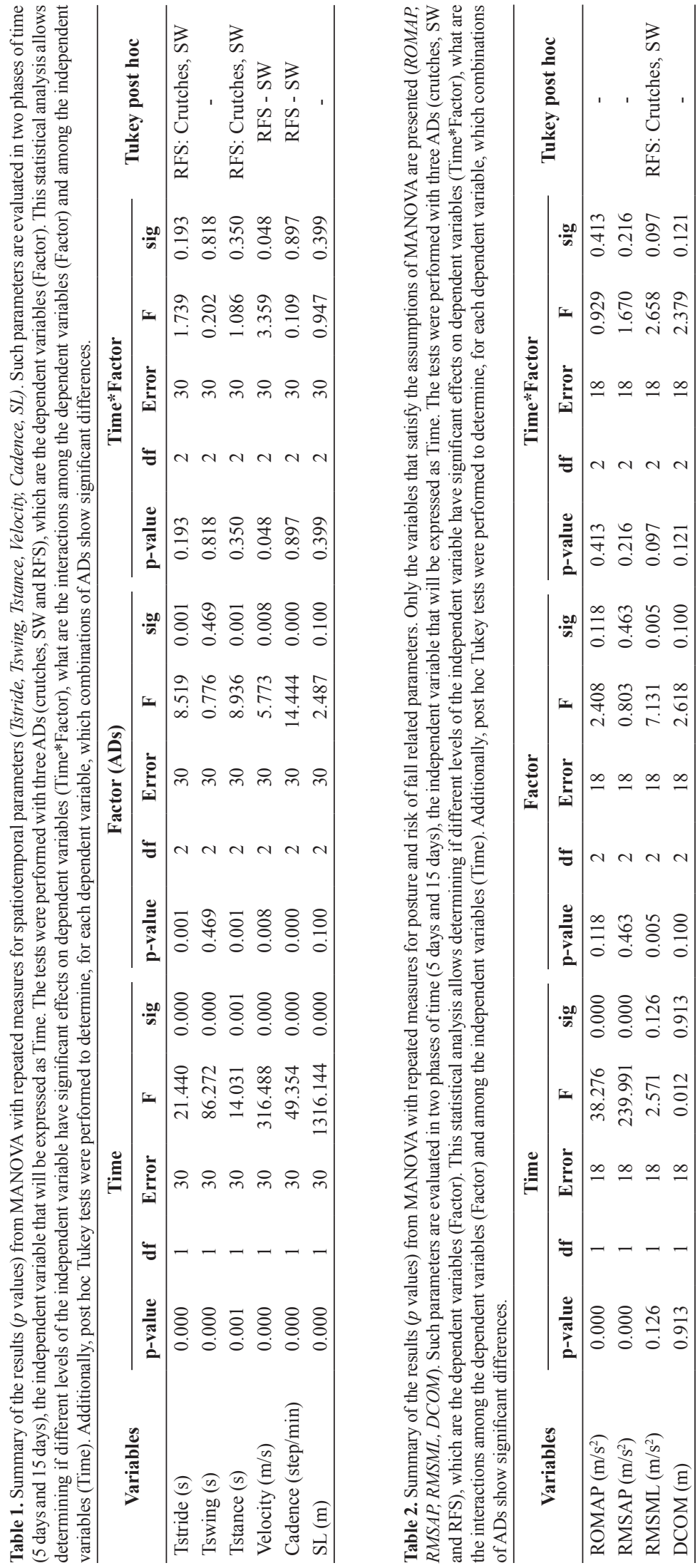


Table 3. Results from the original data (mean \pm standard deviation) for spatiotemporal parameters (Tstride, Tswing, Tstance, Velocity, Cadence, $S L$ for each time phase, 5 and 15 days). Healthy elderly values were extracted from (Hollman et al., 2011) in free walking.

\begin{tabular}{|c|c|c|c|c|}
\hline Parameters & Crutches & SW & RFS & $\begin{array}{c}\text { Healthy elderly (free } \\
\text { walking) }\end{array}$ \\
\hline TStride_5 [s] & $3.555 \pm 1.385$ & $3.264 \pm 1.093$ & $2.043 \pm 0.565$ & \multirow{2}{*}{1.08} \\
\hline TStride_15 [s] & $2.147 \pm 0.624$ & $2.370 \pm 0.773$ & $1.551 \pm 0.599$ & \\
\hline TSwing_5 [s] & $\begin{array}{l}1.152 \pm 0.570 \\
(32.42 \%)\end{array}$ & $\begin{array}{l}1.155 \pm 0.559 \\
(35.40 \%)\end{array}$ & $\begin{array}{l}0.948 \pm 0.385 \\
\quad(46.42 \%)\end{array}$ & \multirow{2}{*}{$\begin{array}{c}0.39 \\
(36.11 \%)\end{array}$} \\
\hline TSwing_15 [s] & $\begin{array}{l}0.793 \pm 0.282 \\
\quad(36.92 \%)\end{array}$ & $\begin{array}{l}0.820 \pm 0.343 \\
\quad(34.60 \%)\end{array}$ & $\begin{array}{l}0.734 \pm 0.241 \\
\quad(47.35 \%)\end{array}$ & \\
\hline TStance_5 [s] & $\begin{array}{l}2.727 \pm 2.046 \\
\quad(67.58 \%)\end{array}$ & $\begin{array}{l}2.092 \pm 0.991 \\
\quad(64.60 \%)\end{array}$ & $\begin{array}{l}1.102 \pm 0.415 \\
\quad(53.58 \%)\end{array}$ & \multirow{2}{*}{$\begin{array}{c}0.69 \\
(63.89 \%)\end{array}$} \\
\hline TStance_15 [s] & $\begin{array}{l}1.164 \pm 0.383 \\
\quad(63.08 \%)\end{array}$ & $\begin{array}{l}1.476 \pm 0.844 \\
(65.40 \%)\end{array}$ & $\begin{array}{l}0.874 \pm 0.430 \\
\quad(52.65 \%)\end{array}$ & \\
\hline Velocity_5 [m/s] & $0.169 \pm 0.102$ & $0.127 \pm 0.074$ & $0.220 \pm 0.066$ & \multirow[b]{2}{*}{1.16} \\
\hline Velocity_15 [m/s] & $0.396 \pm 0.110$ & $0.302 \pm 0.101$ & $0.531 \pm 0.261$ & \\
\hline Cadence_5 [step/min] & $33.466 \pm 11.674$ & $34.170 \pm 10.302$ & $55.132 \pm 17.509$ & \multirow[b]{2}{*}{112} \\
\hline Cadence_15 $[\mathrm{step} / \mathrm{min}]$ & $55.521 \pm 10.899$ & $53.149 \pm 11.029$ & $76.908 \pm 19.607$ & \\
\hline $\mathrm{SL} \_5[\mathrm{~m}]$ & $0.280 \pm 0.074$ & $0.230 \pm 0.086$ & $0.274 \pm 0.075$ & \multirow{2}{*}{0.61} \\
\hline $\mathrm{SL} 15[\mathrm{~m}]$ & $0.422 \pm 0.073$ & $0.344 \pm 0.069$ & $0.398 \pm 0.052$ & \\
\hline
\end{tabular}

Table 4. Results from the original data (meanstandard deviation) for posture and risk of falling parameters (ROMML, ROMAP, RMSML, RMSAP, DCOM for each time phase 5 and 15 days).

\begin{tabular}{|c|c|c|c|c|}
\hline Parameters & Crutches & SW & RFS & $\begin{array}{l}\text { Healthy subjects } \\
\text { (free walking) }\end{array}$ \\
\hline ROM ML_5 [m/ $\left.\mathrm{s}^{2}\right]$ & $0.864 \pm 0.257$ & $0.741 \pm 0.268$ & $0.539 \pm 0.176$ & \multirow{2}{*}{$0.468 \pm 0.218$} \\
\hline ROM ML_15 $\left[\mathrm{m} / \mathrm{s}^{2}\right]$ & $0.445 \pm 0.093$ & $0.444 \pm 0.080$ & $0.978 \pm 0.540$ & \\
\hline ROM AP_5 [m/ $\left.\mathrm{s}^{2}\right]$ & $2.005 \pm 0.859$ & $1.457 \pm 0.536$ & $1.556 \pm 0.907$ & \multirow[b]{2}{*}{$0.447 \pm 0.195$} \\
\hline ROM AP_15 [m/s $\left.\mathrm{s}^{2}\right]$ & $1.833 \pm 0.775$ & $1.081 \pm 0.728$ & $2.365 \pm 1.368$ & \\
\hline RMS AP_ $5\left[\mathrm{~m} / \mathrm{s}^{2}\right]$ & $0.716 \pm 0.162$ & $0.726 \pm 0.252$ & $0.603 \pm 0.213$ & \multirow[b]{2}{*}{$0.316 \pm 0.053$} \\
\hline RMS AP_15 [m/s $\left.\mathrm{s}^{2}\right]$ & $0.649 \pm 0.271$ & $0.434 \pm 0.088$ & $0.589 \pm 0.343$ & \\
\hline RMS ML_5 [m/ $\left./ \mathrm{s}^{2}\right]$ & $0.333 \pm 0.081$ & $0.447 \pm 0.137$ & $0.287 \pm 0.029$ & \multirow{2}{*}{$0.212 \pm 0.004$} \\
\hline RMS ML_15 $\left[\mathrm{m} / \mathrm{s}^{2}\right]$ & $0.408 \pm 0.181$ & $0.318 \pm 0.044$ & $0.232 \pm 0.089$ & \\
\hline DCOM_5 [m] & $1.189 \pm 0.421$ & $0.879 \pm 0.372$ & $0.951 \pm 0.600$ & \multirow{2}{*}{$0.385 \pm 0.001$} \\
\hline DCOM_15 [m] & $0.981 \pm 0.430$ & $0.612 \pm 0.384$ & $1.379 \pm 0.664$ & \\
\hline
\end{tabular}

that the type of gait that the patient presented for each $\mathrm{AD}$ has been decisive for the distinction between the devices based on each parameter.

Regarding the interaction between phase of recovery and the factor (ADs), one verifies that only Velocity is significantly affected by ADs. This result makes sense since one can observe differences of this variable between ADs and between both phases of recovery (Table 3). However, the effect on the other dependent variables is not statistical significant in terms of the interaction. This may mean that the ADs allowed for an effective compensation on these parameters. For TStride, TSwing and TStance, one verifies that RFS presents the lowest differences among phases, being the closest to the reference values (Table 3).
The major differences are associated with crutches (Table 3). For $S L$ and Cadence the SW is associated with the smallest difference, but for these two variables $\mathrm{SW}$ is not the $\mathrm{AD}$ that presents the closest values to the reference values (Table 3 ). In summary, the RFS is associated to the smallest differences between the two phases of recovery. In the first stage the patients are more debilitated, however are still able to walk with the AD. The resultant gait pattern is closer to the controls. This means that RFS provided an adequate support to the patient at his first phase of recovery.

An interesting discussion can be stated with the evolution of TStride, TStance and TSwing. During one gait cycle, $60 \%$ corresponds to stance phase and $40 \%$ to swing phase. For the two phases of recovery, 
the RFS provided the highest percentage in the swing phase and the lowest in the stance phase. The highest percentage in the swing phase may be due to the forearms supports, which provide greater support, decreasing knee pain to patients (Tereso et al., 2014). It is known that an unsecure gait results in an increased stance phase (Kloos et al., 2012). Thus, it is possible that RFS provided an extra support and stability, increasing the sense of security, compared with the other ADs. Regarding SW and Crutches, since they present lower swing phases (lower percentage), they are associated with a slower gait with less security for the user. However, this increase can also be positive because the person dispends more time with the foot on the ground, which means that is feeling less pain.

In terms of Velocity and Cadence, the SW provides the slowest gait, which may be due to the type of movement acquired by this AD. Furthermore, the RFS is associated to the highest values of these two latter parameters (Table 3 (Hollman et al., 2011)). Regarding $S L$, the values are very similar between the ADs, but the one that approaches more to the control is the crutches, perhaps because the recovery phase of the patients was made with the help of this AD.

Inspecting the control values in Table 4, one can see that depending on the patient state, each $\mathrm{AD}$ can influence with a different type of compensation.

\section{Study of posture and risk falling parameters}

By studying the effect of time on the dependent variables (Table 2), one observes that only ROMAP and $R M S A P$ are statistically different in terms of time. It is predicted an improvement of stability with time, which in terms of postural control parameters, is translated into a reduction in $R O M, R M S$ and $D C O M$ (Doheny et al., 2002). By observing Table 4, it appears that, in general, with the progress of time, there was an improvement in the recovery of these patients.

By analysis of Table 2, only RMSML is affected by the factor. In general, for all variables, except for $R M S M L$, it is observed that there are no significant differences between ADs.

Relatively to the interaction results, only $R M S M L$, when influenced by RFS, presents smaller differences between phases and is the closest to control values. Although there is no AD associated with lower differences in the majority of variables, SW is the one with closest values to control, thus providing greater stability for its patients.

Finally, inspecting Table 4, one observes that the greater the acceleration ranges for a specific direction, the greater is the fall risk (Doheny et al., 2002; Huisinga et al., 2011). For the first phase of recovery, RFS presents the lowest values, providing for a greater stability. Since RFS has forearm supports, the patients tend to support their weight on the device. So, it reduces the fall risk for its patients that, at this phase, present a very unstable gait. At 15 days, $\mathrm{SW}$ is the one that provides for a better stability in comparison with the other ADs. This result may be due to the erect posture that the patient presents while using SW, as well as the static support (fixed and wide base) that is given by this device. With these advantages, a low trunk oscillation is observed in all directions. At 15 days, RFS provides for a lower stability. This can be explained by the fact that the patients no longer require as much support in the second phase. The decrease of load on the RFS can increase the trunk oscillation, given the continuous and faster movement. Therefore, this leads to the conclusion that it might be positive to use this AD only in an initial phase of rehabilitation and with patients with a lower degree of instability. Observing Crutches results, one concludes that this $\mathrm{AD}$ is not associated with a good stability nor fall-risk, in both phases.

On the overall, SW is good to give stability to the patient, however, RFS allows the patient to present a gait pattern closest to a natural gait.

In conclusion, there are differences between the gait parameters of TKA patients depending on the device used, mainly between the RFS and the others. This divergence between the ADs may be due to the type of motion pattern presented while using each AD. When feeling discomfort on the operated leg, the patient may feel the need to overcompensate its movement, by changing its pattern. In general, it was found that the RFS provides a proper support and functional compensation on the first phase of recovery, because even in a debilitated condition, patients are able to walk with the assistance of this device with a more natural and faster gait pattern. In contrast, SW tends to induce a slower gait. With these results, patients that are recovering from TKA surgery should use RFS during the recovery in order to present a more natural gait, not putting unnecessary effort on the non-operated leg.

From the postural and fall risk parameters it is possible to conclude that there is a reduction on the fall-risk and an increase in stability between both phases of recovery in all ADs. Thus, there is a progression of the state of recovery of the patients. On the overall, $\mathrm{SW}$ provides for a more stable gait. This means that if the patient presents a more debilitated state in terms of stability for walking, the SW device should be prescribed.

Therefore, depending on the state of recovery of the patient, different $\mathrm{AD}$ should be prescribed. This conclusion is very important and it was achieved 
through the observation of the different variables calculated on this study among different time phases and devices. So, it was possible to verify that it is necessary to revise some of the clinical and rehabilitation indications for the recovery of these patients.

\section{Acknowledgements}

Work supported by the Portuguese Science Foundation (grant SFRH/BD/76097/2011).

\section{References}

Braddom RL. Physical medicine \& rehabilitation. 3rd ed. Philadelphia: Elsevier Health Sciences; 2006. p. 1504.

Bradley SM, Hernandez CR. Geriatric assistive devices. American Family Physician. 2011; 84(4):405-11. PMid:21842786.

Debi R, Mor A, Segal G, Segal O, Agar G, Debbi E, Halperin N, Haim A, Elbaz A. Correlation between single limb support phase and self-evaluation questionnaires in knee osteoarthritis populations. Disability and Rehabilitation. 2011; 33(13-14):1103-9. http://dx.doi.org/10.3109/09638 288.2010.520805. PMid:21208029.

Doheny EP, McGrath D, Greene BR, Walsh L, McKeown D, Cunningham C, Crosby L, Kenny RA, Caulfield B. Displacement of centre of mass during quiet standing assessed using accelerometry in older fallers and non-fallers. In: Conference Proceedings Annual International Conference IEEE Engineering Medical \& Biology Society; 2012; San Diego, CA. IEEE; 2002. p. 3300-3.

Fernandes V. Desenvolvimento de um sistema para análise de equilibrio baseado em sensores inerciais [dissertation]. Braga: Universidade do Minho; 2013.

Henriksen M, Lund H, Moe-Nilssen R, Bliddal H, DanneskiodSamsøe B. Test-retest reliability of trunk accelerometric gait analysis. Gait \& Posture. 2004; 19(3):288-97. http://dx.doi. org/10.1016/S0966-6362(03)00069-9. PMid:15125918.

Hollman JH, McDade EM, Petersen RC. Normative spatiotemporal gait parameters in older adults. Gait \& Posture. 2011; 34(1):111-8. http://dx.doi.org/10.1016/j. gaitpost.2011.03.024. PMid:21531139.

Huisinga JM, George RJS, Horak FB. Variability measures of trunk and pelvis acceleration during walking and quiet stance are related in patients with multiple sclerosis and in healthy controls. In: The American Society of Biomechanics Annual Meeting; 2011; Long Beach, CA. ASB; 2011. p. 1-2.

Ishikura T. Biomechanical analysis of weight bearing force and muscle activation levels in the lower extremities during gait with a walker. Acta Medica Okayama. 2001; 55(2):7382. PMid:11332202.

Kegelmeyer DA, Parthasarathy S, Kostyk SK, White SE, Kloos AD. Assistive devices alter gait patterns in Parkinson disease: advantages of the four-wheeled walker. Gait \& Posture. 2013; 38(1):20-4. http://dx.doi.org/10.1016/j. gaitpost.2012.10.027. PMid:23237981.
Kloos AD, Kegelmeyer DA, White SE, Kostyk SK. The impact of different types of assistive devices on gait measures and safety in Huntington's disease. PLoS One. 2012; 7(2):e30903. http://dx.doi.org/10.1371/journal. pone.0030903. PMid:22363511.

Lee J-A, Cho SH, Lee Y-J, Yang H-K, Lee J-W. Portable activity monitoring system for temporal parameters of gait cycles. Journal of Medical Systems. 2010; 34(5):959-66. http:// dx.doi.org/10.1007/s10916-009-9311-8. PMid:20703612.

Liu HH, McGee M, Wang W, Persson M. Comparison of gait characteristics between older rolling walker users and older potential walker users. Archives of Gerontology and Geriatrics. 2009; 48(3):276-80. http://dx.doi.org/10.1016/j. archger.2008.02.004. PMid:18359111.

Liu HH. Assessment of rolling walkers used by older adults in senior-living communities. Geriatrics \& Gerontology International. 2009; 9(2):124-30. http://dx.doi.org/10.1111/ j.1447-0594.2008.00497.x. PMid:19740354.

Martins M, Santos CP, Costa L, Frizera A. Multivariate analysis of walker-assisted ambulation. In: IEEE 3rd Portugal Meeting Bioengineering (ENBENG); 2013; Braga, Portugal. IEEE; 2013. p. 23-6.

Melis EH, Torres-Moreno R, Barbeau H, Lemaire ED. Analysis of assisted-gait characteristics in persons with incomplete spinal cord injury. Spinal Cord. 1999; 37(6):430-9. http://dx.doi.org/10.1038/sj.sc.3100850. PMid:10432263.

Priebe JR, Kram R. Why is walker-assisted gait metabolically expensive? Gait \& Posture. 2011; 34(2):265-9. http://dx.doi. org/10.1016/j.gaitpost.2011.05.011. PMid:21665475.

Roomi J, Yohannes AM, Connolly MJ. The effect of walking aids on exercise capacity and oxygenation in elderly patients with chronic obstructive pulmonary disease. Age and Ageing. 1998; 27(6):703-6. http://dx.doi.org/10.1093/ ageing/27.6.703. PMid:10408664.

Sabatini AM, Martelloni C, Scapellato S, Cavallo F. Assessment of walking features from foot inertial sensing. IEEE Transactions on Biomedical Engineering. 2005; 52(3):486-94. http://dx.doi.org/10.1109/TBME.2004.840727. PMid:15759579.

Smidt GL, Mommens MA. System of reporting and comparing influence of ambulatory aids on gait. Physical Therapy. 1980; 60(5):551-8. PMid:7384225.

Tereso A, Martins M, Santos CP, Vieira da Silva M, Gonçalves L, Rocha L. Detection of gait events and assessment of fall risk using accelerometers in assisted gait. In: Proceedings 11th International Conference Informatics Control and Automation Robotics; 2014; Vienna, Austria. IEEE; 2014. p. 788-93.

Tung JY, Gage WH, Poupart P, McIlroy WE. Upper limb contributions to frontal plane balance control in rollatorassisted walking. Assistive Technology. 2014; 26(1):15-21, quiz 22-3. http://dx.doi.org/10.1080/10400435.2013.7894 56. PMid:24800450.

Van Hook FW, Demonbreun D, Weiss BD. Ambulatory devices for chronic gait disorders in the elderly. American Family Physician. 2003; 67(8):1717-24. PMid:12725450. 
Vogt L, Lucki K, Bach M, Banzer W. Rollator use and functional outcome of geriatric rehabilitation. Journal of Rehabilitation Research and Development. 2010; 47(2):151-6. http://dx.doi.org/10.1682/JRRD.2009.09.0148. PMid:20593328.

Wellmon R, Pezzillo K, Eichhorn G, Lockhart W, Morris J. Changes in dual-task voice reaction time among elders who use assistive devices. Journal of Geriatric Physical Therapy.
2006; 29(2):74-80. http://dx.doi.org/10.1519/00139143200608000-00006. PMid:16914064.

Youdas JW, Kotajarvi BJ, Padgett DJ, Kaufman KR. Partial weight-bearing gait using conventional assistive devices. Archives of Physical Medicine and Rehabilitation. 2005; 86(3):394-8. http://dx.doi.org/10.1016/j.apmr.2004.03.026. PMid:15759217.

\section{Authors}

